\title{
TActical Sensor network TEst bed (TASTE)
}

\author{
Drs. Ir. Ph. van Dorp ${ }^{1}$, Dr. H.H.P.Th. Bekman ${ }^{1}$ and Ing. R.D.J. Sandbrink ${ }^{1}$ \\ ${ }^{1}$ TNO Defence, Security and Safety \\ The Hague, The Netherlands \\ www.tno.nl
}

\begin{abstract}
TASTE is a software tool for specifying and deploying unattended ground sensors (UGS) in a composition which the commander assumes will suit his needs the best. With TASTE different sensor types such as acoustic, magnetic, seismic, radar and IR imaging sensors can be deployed virtually and their individual and combined performances analyzed. Sensors can be deployed in scenarios for neutral, friendly and enemy movements and a set of typical environmental conditions can be selected as input to the simulator. TASTE will play user defined scenario, calculating and monitoring the performance and behavior of the individual UGS sensors or of the entire sensor network.
\end{abstract}

\section{Keywords}

Sensor network, simulation model, UGS, radar, IR camera

\section{INTRODUCTION}

Sensor fusion combines information of individual sensors in such way that the combined performance is expected to be better than the individual sensor performance. This is not only the case for different sensor types but also for several similar sensors located at different positions. To determine the performance of these sensor networks in a real environment is not an easy task. Therefore a simulation environment can be used to estimate these performances.

A few years ago TNO started to design a "TActical Sensor network Test bed" (TASTE), as

commissioned by the Royal Netherlands Army, to give them a better understanding of the performance of different types of unattended ground sensors separate or within a network (which will become more common in the future).

The developments in the ICT area during the nineties, generated the idea of using UGS in a network which would enable the combination of information from different sensors at different locations in real time. References [4] and [8] give study results about these topics. Thus, by using the whole network as a sensor, one may expect that the built-in redundancy of a sensor network will be utilized far more efficiently than exploiting the sensors in their autonomous modes. This approach will result in a sensor network with increased reliability and lower unwanted alarm rates in different and more difficult environmental circumstances.

Section 2 describes the TASTE setup.

Section 3 describes the theory and algorithm used for each of the different implemented sensor types (UGS, radar and IR imaging).

Results of an example simulation are presented in section 4 .

The last section gives the conclusions.

Unmanned/Unattended Sensors and Sensor Networks V, edited by Edward M. Carapezza Proc. of SPIE Vol. 7112, 711206 · C 2008 SPIE · CCC code: 0277-786X/08/\$18 · doi: 10.1117/12.802138 


\section{TASTE SETUP}

Before running TASTE, the user has a choice to:

- define, compose and deploy an appropriate sensor suite (consisting of different sensor types and configurations);

- compose a tactical military scenario, including the targets, routes, time-aspects, etc;

- define environmental conditions (e.g. precipitation, temperature, wind gradient, terrain conditions, etc);

- define additional features (e.g. percentage of sensor loss, malfunctioning equipment, etc).

As soon as the simulation starts, TASTE shows on a map what really happens according to the chosen scenario (ground truth) and in a separate window what the sensors make of it.

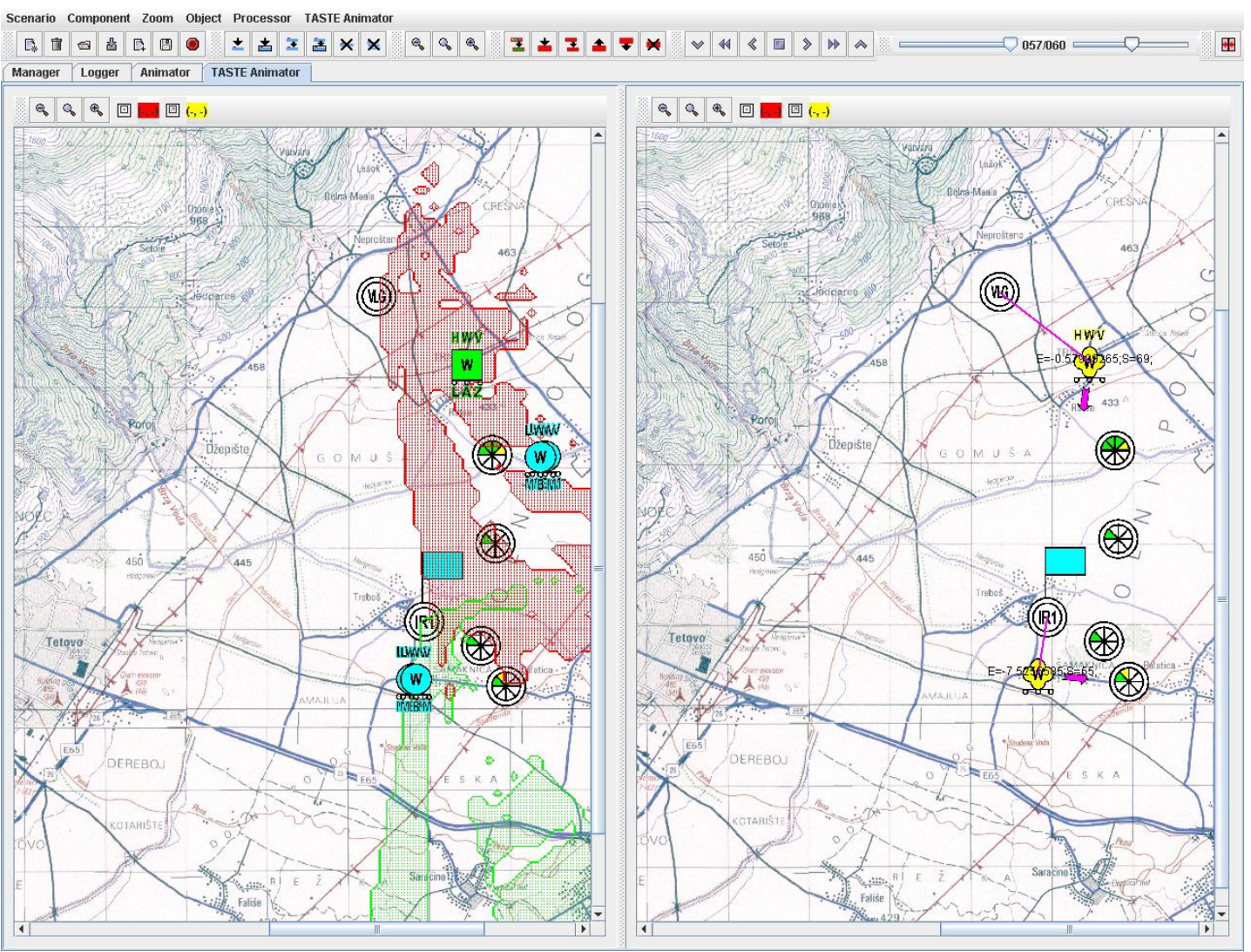

Fig.1. Fragment of a TASTE simulation result; left: ground truth; right: actual awareness through the chosen sensors.

As shown in figure 1 the simulation results are presented as an animation which is divided in two panes. The left pane displays the ground truth scenario on a map including sensor deployment, moving targets, radar coverage (red shade) and IR camera coverage (green shade). The right pane displays the

sensors detections on the same map including sensor deployment. The UGS are presented as partitioned colored circles. These colors stand for the type of UGS (green stands for a seismic acoustic sensor; yellow for a magnetic sensor; red for a IR sensor; three green triangles stands for a seismic acoustic array; and a combination of different types is also possible). Depending on the type of sensor, detections in the right pane are presented differently. For example a radar and IR camera can present a bearing and distance there where for simple UGS only a detection symbol is presented. The colored targets and detections stand for neutral (green), friendly (blue), hostile (red) and unknown (yellow). 


\section{SENSORS}

\subsection{UNATTENDED GROUND SENSOR}

\section{Introduction}

Rather than being based upon complicated acoustic, seismic and EM propagation models (ref. [1], [2], [5], [6] ), TASTE uses best guesses and approximations according to a number of rules of thumb. This approach guarantees a good overall impression of UGS performance and a good view of the coverage and performance of the sensor suite given the defined scenario, etc. It also will teach TASTE users the best way of composing and sizing the sensor suite, given the military tasks to be accomplished and depending upon the existing environmental conditions.

The most commonly used UGS in TASTE are combinations of acoustic, seismic, magnetic and IR-line sensors, the performance of which is based upon commercially available UGS. Because small radars and IR-camera's are expected to be used more often in UGS in the future, these sensors have also been incorporated into TASTE.

\section{UGS algorithm}

The UGS algorithm is based upon the idea that a sensor has a maximum detection range for each specific target it can detect. Especially the main influences on UGS (e.g. weather, terrain, time of day, target speed, other targets nearby) will decrease the detection range for a specific target at a specific moment. See figure below.

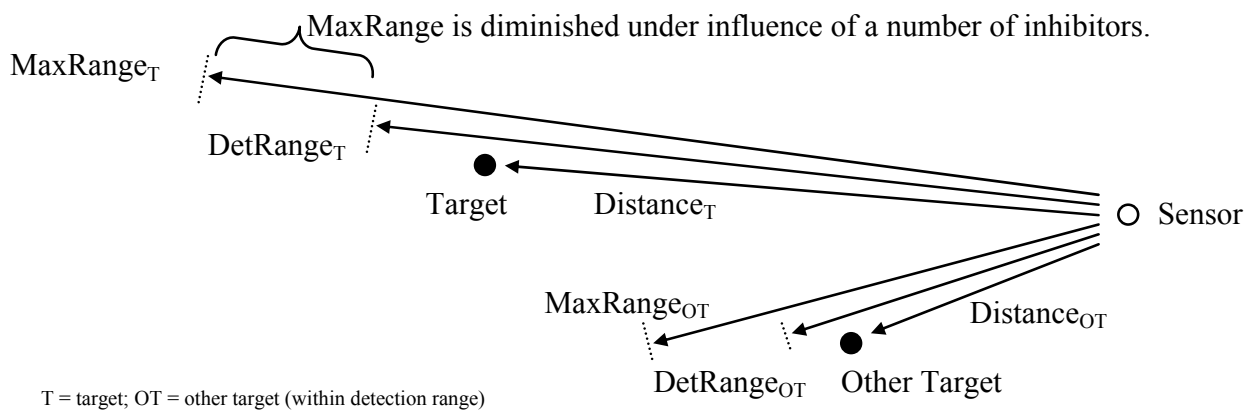

Fig.2. UGS algorithm aspects in TASTE.

The first step in the algorithm is to find out if the sensor is able to detect the target at all. These sensortarget relations are assigned as tables in TASTE. If there is no relation assigned between the sensor and the target the next steps in the algorithm will be ignored, and no detection will appear. Otherwise (if there is a relation between sensor and target) the target must be in range of the sensor's maximum detection range for this target. If so, all influences (including influences from other targets (OT) within range of the sensor) which are for that moment relevant in the scenario are cumulated and will diminish the sensor's detection range. If at this moment the target's distance is still within detection range a simple distance loss is calculated depending on the sensor-target distance. Next step is to take the detection rate for this sensor-target relation (which is also assigned in TASTE tables). Together with the distance loss, the cumulated influences and a random draw the target is detected or not. See formula below.

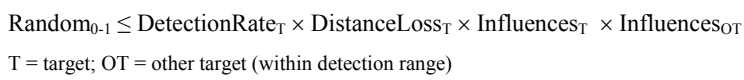


The next steps are to generate the relevant sensor output values. Not each type of UGS is able to calculate target locations, distances, speeds or what ever they are capable of. Also these relations between the sensor types (acoustic, magnetic, seismic, etc.) and sensor outputs (distance, azimuth, direction, speed, elevation, counting) are assigned as accuracy values in the TASTE tables. Depending on these assigned relations the output is calculated by creating a random offset on the actual target values which are available in the scenario. This random offset is based on again the influences and the target-sensor distance together with the accuracy value of the sensor output value. See formula below for an example of the speed output value.

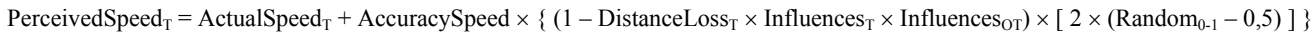

$\mathrm{T}=$ target; $\mathrm{OT}=$ other target (within detection range)

The TASTE test bed has also the option to simulate the sensor's performance as separate point to point (sensor to command post) connection or as if all sensors in the scenario were operating in one communication network. In this network mode all sensors also act as relay units (i.e. with the ability to relay detections through the network to the command post). Depending on the selected option and the transmission distances between the sensors and the command post a detection transmission is able to find its way through the network to the command post. Transmission influences are not yet implemented in TASTE. At this moment a partial study is running to find the main aspects of communication in sensor networks which will be implemented in the TASTE test bed.

To display the performance of different sensor types within TASTE not only the actual targets in the scenario are to be taken into account. Also the false alarms should be calculated and displayed during the scenario running to create a truthful situation awareness picture. During the simulation in TASTE for each sensor is determined if a false alarm should be generated. For each classification possibility of the sensor only the inhibitors (ground type, precipitation, wind) at the sensor's location are taken into account which could affect a false alarm. A schedule is made up out of these parameters to randomly draw a false alarm target classification. The false alarm rate in the TASTE tables is defined as the maximum number of false alarms a sensor produces per 24 hours. Besides the created target classification for the false alarm also the relevant sensor output values are created as if it was a real target.

\subsection{RADAR}

\section{Introduction}

To compare the performance of unattended ground sensors with radar, TNO started two years ago with the integration of a radar algorithm in TASTE. Because of the more detailed algorithm which should be used, it was necessary to create a link with the modular advanced radar simulator (MARS) also developed at TNO. General radar theory used in MARS is given by Skolnik ref. [11] and Stimson ref. [12]. An introduction to radar simulation algorithms used in MARS gives Mitchell ref. [13]. A TASTE scenario is first simulated in MARS to gain specific input data for every used radar in the scenario. Because of the Line of Sight (LOS) restrictions for radar "Rest Heights" (minimum height at which the radar can see a target/object) are calculated with MARS (see figure 3) using a digital elevation map of the TASTE scenario, and a radar coverage picture is created to fit in the ground truth view of TASTE. After simulation in TASTE, the output shows the ground truth scenario with the radar coverage picture in the left view and the sensor's perception (radar and UGS) of the scenario in the right view of TASTE. 


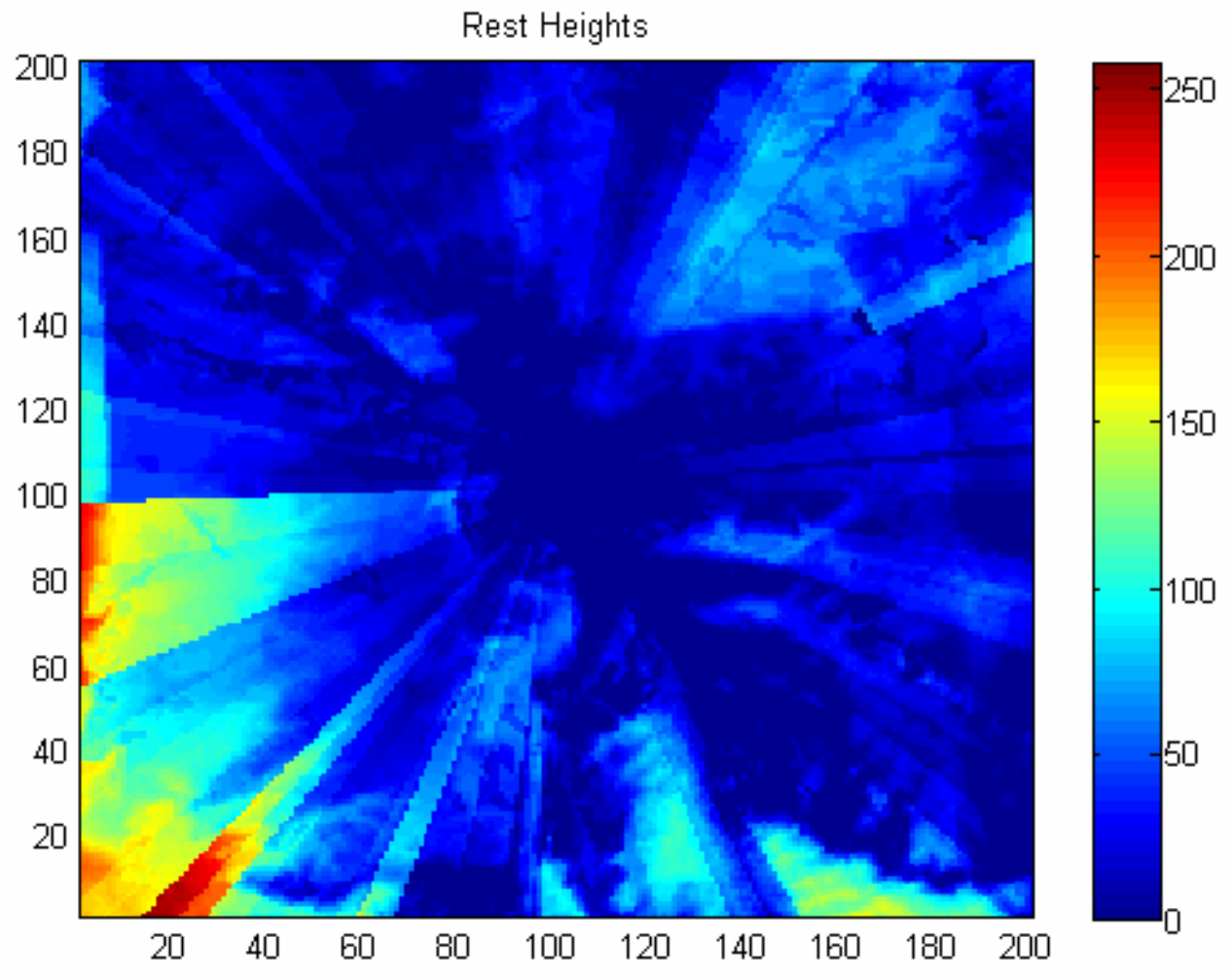

Fig.3. MARS Rest Heights for one specific radar location in a specific scenario.

\section{Radar algorithm}

The radar algorithm in TASTE starts with determining the target's position with regard to the radar position (range \& cross range). In the next step the pre-calculated rest heights and target height are used to determine if the radar is in line of sight (LOS) with the target. If there is no LOS the detection rate is 0 and no detection will appear. Otherwise if there is LOS between radar and target a radar cross section (RCS) ground correction is calculated using the target angle, tangential speed and if present the ground clutter. The ground clutter is also available in TASTE via a pre-calculation in MARS, and is an array of data with $\mathrm{dB}$ proportions of ground clutter power and radar noise power. If there is no ground clutter present, the RCS ground correction is 0 . At last the probability of detection (POD) is determined by using a MARS pre-calculated free-space detection probability array together with the target RCS, the target-radar distance and, if present, the RCS ground correction (see figure 4). 


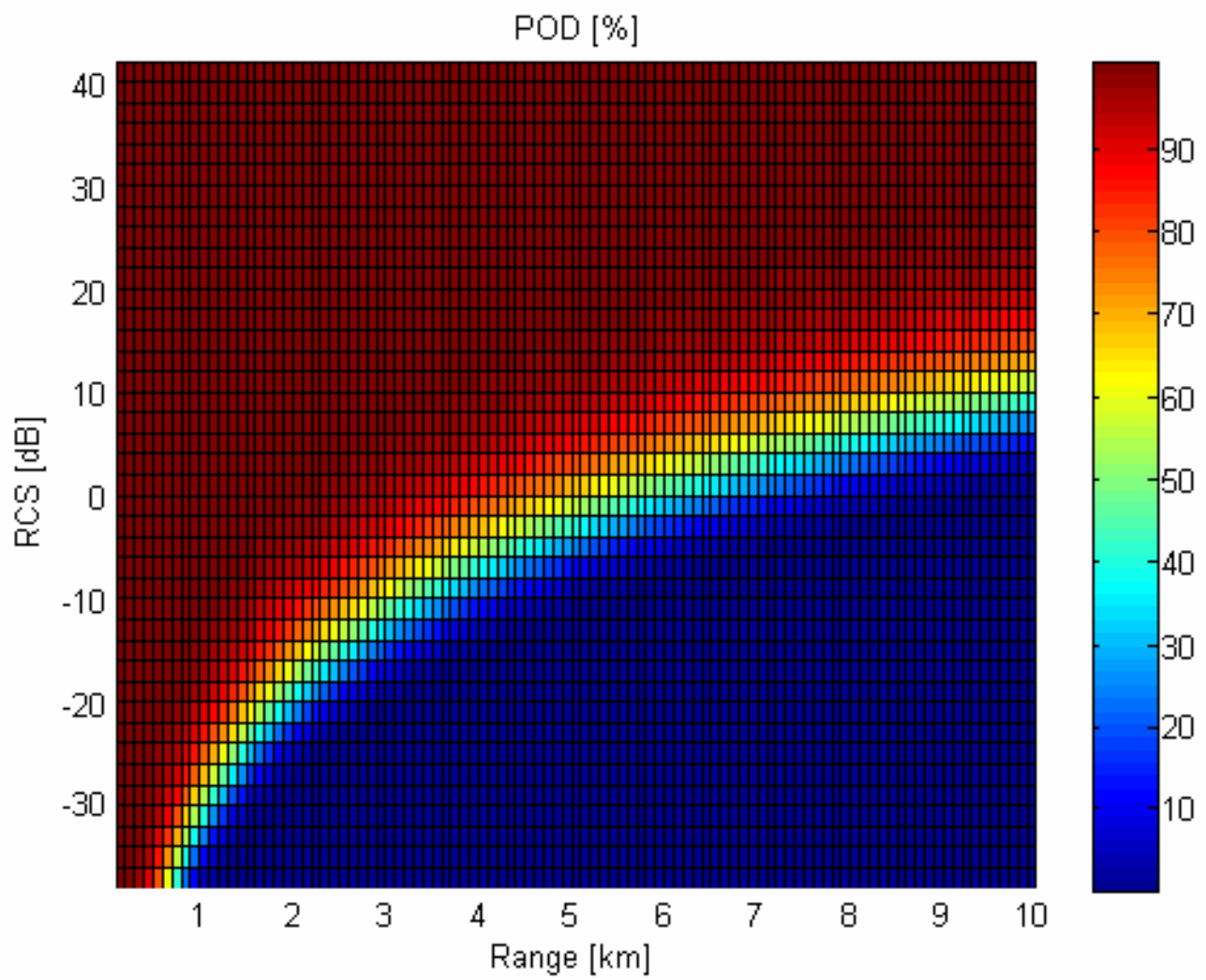

Fig.4. Example of a free-space detection probability array.

The above algorithm suffices for target tracking radars. In case of surveillance radars with a fixed antenna elevation pattern, the calculation of the detection rate is slightly different. The RCS ground clutter correction is calculated without using the target elevation angle. The target RCS is corrected according to the extent the target is within the antenna beam. In TASTE a table with RCS values is defined as a function of target type and radar type. Because the RCS value for every target depends also on the used radar frequency, a correction is also taken into account.

To examine the radar detections in the right pane of a TASTE simulation output, the radar coverage is presented in the left pane as a red shade overlay on the map. See figure 1.

\subsection{IR CAMERA}

\section{Introduction}

UGS could nowadays have integrated IR-camera's, so TNO has also implemented separate human operated IR-camera sensors within TASTE. This makes it possible to compare the performance of IRcamera's with (networked) UGS. Within the TASTE environment a number of IR cameras can be placed. The cameras are defined in terms of its field of view (FOV), its spatial resolution and its MRTD (minimum resolvable temperature difference) as a function of spatial frequency. Ref. [9] and [10] gives specific information about MRTD. The detection, recognition and identification (DRI) probability of a target is then calculated. The DRI probability is a function of the apparent temperature differences between target and background, the target size and the target range. Furthermore the apparent temperatures depend on weather, time of day and engine state. The sensor background is created using line of sight calculations in combination with a digital elevation map and a terrain classification map. Figure 5 shows a typical sensor background image. 


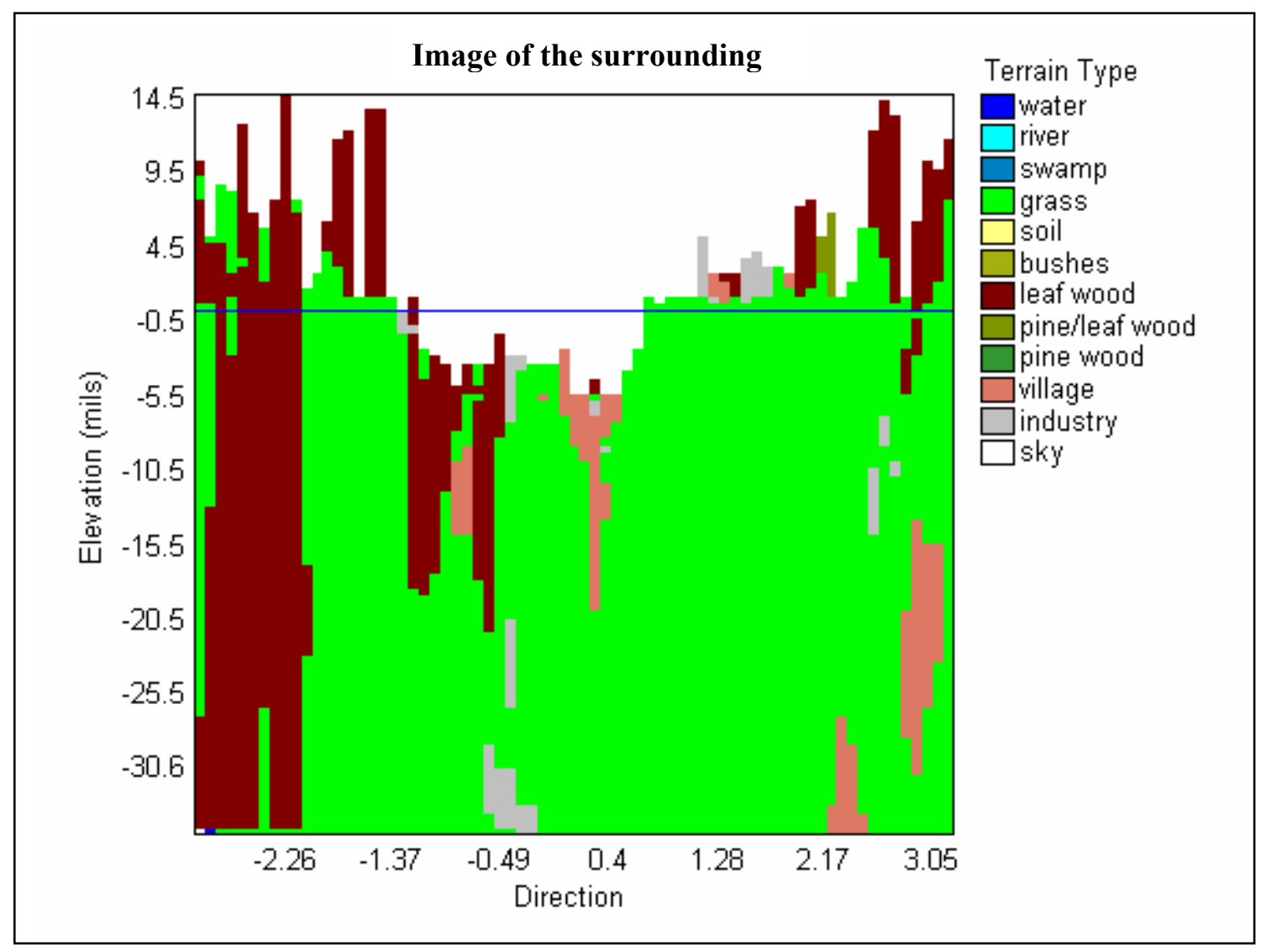

Fig.5. Surrounding image for one specific IR-camera location.

\section{IR camera algorithm}

Like the radar algorithm, the IR algorithm in TASTE starts with determining the target's position with regard to the IR camera position. In the next step the calculated rest heights in MARS are used together with the target height to determine if the sensor has line of sight (LOS) with the target. If there is no LOS the detection rate is 0 and no detection will appear. Otherwise if there is LOS the background image of the IR camera is determined by using the calculated surrounding image in MARS (See figure 5) and the IR camera settings (bearing and field of view) as defined in the TASTE scenario. Depending on scenario settings as timeframe (day or night), atmospheric conditions (clear sky, overcast, light rain, medium rain and heavy rain) and target state (engine off, engine idle, engine on), the IR temperatures (Kelvin) of the background and the target is calculated. These IR temperatures are stored in TASTE as shown in tables $6 \& 7$. 


\begin{tabular}{|l|l|l|l|l|l|l|l|l|l|l|l|}
\hline \multicolumn{1}{|c|}{} & \multicolumn{1}{c|}{ CS =Clear Sky; OV = Overcast: LR = Light Rain; MR = Moderate Rain; HR = Heavy Rain } \\
\hline nr. & atmosphere: & CS & OV & LR & MR & HR & CS & OV & LR & MR & HR \\
\hline 11 & water & 10.9 & 12.1 & 12.1 & 12.1 & 12.1 & 10.8 & 12.1 & 12.1 & 12.1 & 12.1 \\
\hline 10 & river & 10.9 & 12.1 & 12.1 & 12.1 & 12.1 & 10.8 & 12.1 & 12.1 & 12.1 & 12.1 \\
\hline 9 & swamp & 21.2 & 15.5 & 7.5 & 5.6 & 4.7 & 7.8 & 9.1 & 5.2 & 4.4 & 4.1 \\
\hline 8 & grass & 20.1 & 15.1 & 7.5 & 5.6 & 4.8 & 8.2 & 8.5 & 5.4 & 4.5 & 4.2 \\
\hline 7 & soil & 34.1 & 20.7 & 13.5 & 9.5 & 7.2 & 3.9 & 5.2 & 4.5 & 4.2 & 4.0 \\
\hline 6 & bushes & 20.1 & 15.1 & 7.5 & 5.6 & 4.8 & 8.2 & 9.5 & 5.4 & 4.5 & 4.2 \\
\hline 5 & leaf wood & 20.1 & 15.1 & 7.5 & 5.6 & 4.8 & 8.2 & 9.5 & 5.4 & 4.5 & 4.2 \\
\hline 4 & pine-leaf wood & 20.1 & 15.1 & 7.5 & 5.6 & 4.8 & 8.2 & 9.5 & 5.4 & 4.5 & 4.2 \\
\hline 3 & pine wood & 20.1 & 15.1 & 7.5 & 5.6 & 4.8 & 8.2 & 9.5 & 5.4 & 4.5 & 4.2 \\
\hline 2 & village & 64.9 & 33 & 15.9 & 10.1 & 7.3 & 1.3 & 2.6 & 3.3 & 3.5 & 3.6 \\
\hline 1 & industrial & 64.9 & 33 & 15.9 & 10.1 & 7.3 & 1.3 & 2.6 & 3.3 & 3.5 & 3.6 \\
\hline 0 & sky & 21 & 20 & 20 & 20 & 20 & 19 & 20 & 20 & 20 & 20 \\
\hline
\end{tabular}

Table 6. Background IR temperatures (Kelvin).

\begin{tabular}{|l|l|l|l|l|l|l|l|l|l|l|l|}
\hline \multicolumn{1}{|c|}{ CS = Clear Sky; OV = Overcast; LR = Light Rain; $\mathrm{MR}=$ Moderate Rain; HR = Heavy Rain } \\
\hline Personal carrier (YPR) & \multicolumn{1}{|c|}{ Day } & \multicolumn{4}{c|}{ Night } \\
\hline atmosphere: & CS & OV & LR & MR & HR & CS & OV & LR & MR & HR \\
\hline Engine off & 50.8 & 29.8 & 26.9 & 25.4 & 24.6 & 3.9 & 4.6 & 4.3 & 4.2 & 4.1 \\
\hline Engine idle & 54.7 & 34.6 & 31.9 & 30.4 & 29.6 & 10.0 & 10.7 & 10.4 & 10.3 & 10.2 \\
\hline Engine on & 70.9 & 53.7 & 51.5 & 50.3 & 49.6 & 33.7 & 34.2 & 34.0 & 33.9 & 33.9 \\
\hline
\end{tabular}

Table 7. IR temperatures (Kelvin) for a Personal carrier vehicle (YPR) at different conditions.

The final step is to calculate the detection rate of the IR camera using specific sensor sensitivity (MRTD), the IR temperature difference between target and background, target dimension, atmospheric conditions and target-sensor distance.

To examine the IR camera detections in the right pane of a TASTE simulation output, the IR camera coverage is presented in the left pane as a green shade overlay on the map. See figure 1.

\section{SIMULATION RESULTS}

Based on simple military scenarios several simulations with one or more different sensors under different conditions (influences) were carried out to initially define all sensor specifications in TASTE tables. To validate TASTE performance output was compared with field trial data available at TNO ref. [3] and from data collections of NATO field campaigns ref. [7]. Besides the performance output being displayed on a map (see figure 1) a data array is also generated. This data array consists of a number of key variable values calculated for each sensor-target combination at each time step during the scenario run. With this data it's possible to examine the simulation in, for example Excel, and create diagrams as presented in figure 8 below.

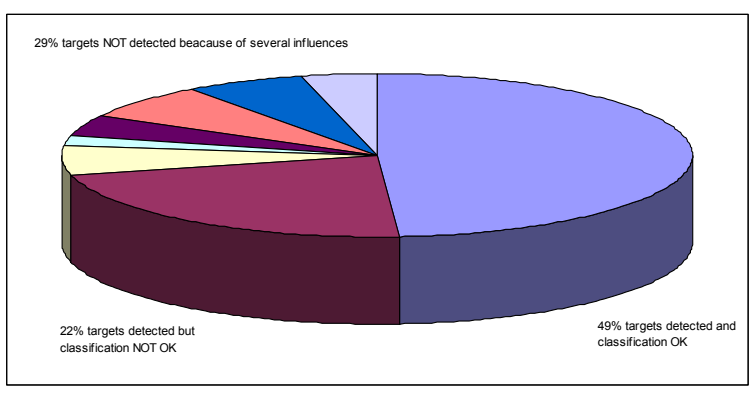

Fig.8. Example of simulated sensor performance in TASTE. 


\section{CONCLUSION}

The base principle of the TASTE algorithm and its simulation results gives a sufficient overall idea of sensor performance. The implemented UGS algorithm is far from complex but several important aspects are taken into account. The implemented radar and IR camera sensors algorithm is more detailed as a result of their complex nature and sensor influences.

The simulation results are comparable with field trials and confirm that TASTE can be used for UGS performance evaluation.

More validation of TASTE output is necessary for which more field trial data should be obtained. Advances in UGS technology and performance and the appearance of new target types also require continual updating and validation of TASTE.

\section{REFERENCES}

[1] K. Attenborough (UK), S. Taherzadeh (UK), H. Bass (USA), R. Raspet (USA), G. Becker (Germany), A. Güdesen (Germany), A. Chrestman (USA), G. Daigle (CA), A. L'Espérance (CA), Y. Gabillet (France), K. Gilbert (USA), Y. Li (USA), M. White (USA), P. Naz (France), J. Noble (USA), H. van Hoof (Netherlands); "Benchmark cases for outdoor sound propagation models", Journal of acoustical society of America (JASA) 97(1), pp 173-191, (1995).

[2] Ir. H.A.J.M. van Hoof, "Outdoor sound propagation, its implications to unattended ground sensors", TNO report, (1986).

[3] Henkel, R.J.G., Hoof, H.A.J.M. van, "Experiments with acoustic and seismic classification of vehicles", IR-1987-32, (1987).

[4] Ir. H.A.J.M. van Hoof, O.E. Slichtenbree, "Onbemande grondsensors", Militaire Spectator 7, pp 320-325 (1988).

[5] Ir. H.A.J.M. van Hoof, "Helikopterdetectie met akoestische middelen", Militaire Spectator 5, pp 226-230 (1989).

[6] Ir. H.A.J.M. van Hoof, “Acoustic helicopter detection and recognition”, Proceedings Shape Technical Center, NIS workshop, The Hague, (1989).

[7] Data collection field campaigns, organised and executed by members of the NATO AC/243 Panel 3 RSG-11 in Munster (Germany, 1980), Schweinfurt (Germany 1987), Dreux (France, 1988), Bourges (France, 2002).

[8] Numerous presentations, given during the SPIE conferences, and published in: E. M. Carapezza, Proceedings SPIE, Unattended Ground Sensor Technologies and Applications (2001-2007),

E. M. Carapezza, Proceedings SPIE, Sensors and Command, Control, Communications, and Intelligence Technologies for Homeland Defense and Law Enforcement (2001-2007).

[9] A.N. de Jong, E.M. Franken, H. Winkel, "Alternative measurement techniques for infrared sensor performance", Optical Engineering Vol 42, Issue 3, pp 712-724 (2003).

[10] P. Bijl, J.M. Valeton, "TOD, A new method to characterize electro-optical system performance", Proceedings SPIE, Vol 3377, pp 182-193 (1998).

[11] Merrill I. Skolnik, "Introduction to Radar Systems", second edition 1980.

[12] George W. Stimson, "Introduction to Airborne Radar", second edition 1998.

[13] Richard L. Mitchell, "Radar Signal Simulation”, 1976. 